\title{
CHARISMA: A Novel Channel-Adaptive TDMA-Based Multiple Access Control Protocol for Integrated Wireless Voice and Data Services
}

\author{
Vincent K. N. Lau and Yu-Kwong Kwok \\ Email: \{knlau,ykwok\}@eee.hku.hk \\ Department of Electrical and Electronic Engineering \\ The University of Hong Kong, Pokfulam Road, Hong Kong \\ Tel: +852-2857-8483, Fax: +852-2559-8738 \\ Corresponding Author: Vincent K. N. Lau
}

\begin{abstract}
In this paper ${ }^{1}$, we introduce a novel multiple access control (MAC) protocol for integrated wireless voice and data services on the uplink channel in a cellular wireless network. The proposed protocol is TDMA based and the uplink frame is divided into two subframes: a request subframe and an information subframe. Our scheme, called CHARISMA (Channel Adaptive Reservation-based Isochronous Multiple Access), works by first gathering users' request via the mini-slots in the request subframe and then decides on the allocation of the information slots in the information subframe based on the channel states ranking of the mobile users. Our extensive simulation results indicate that significant improvements in terms of throughput, delay, and packet loss probability are achieved using the CHARISMA protocol.
\end{abstract}

\section{INTRODUCTION}

In general, for a wireless communication system with integrated voice and data services [1], previous multiple access control (MAC) protocols attempt to accommodate more data users, which do not impose constraints on data delay, by exploiting the silence gaps of the voice users, which require boundeddelay packet transmission and hence, enjoy a higher transmission priority than data users in that reservation is allowed for the former but not the latter. However, while sophisticated slot assignment strategies with articulated frame structures are proposed in these methods, none of them considers the effect of burst channel errors on protocol performance, let alone the investigation of exploiting the error characteristics to enhance performance. Essentially, these previous protocols are designed and analyzed based on the assumption that packet transmission through

\footnotetext{
${ }^{1}$ This research was jointly supported by research initiation grants from the HKU CRCG under contract numbers 10202523 and 10202518 , and by a grant from the Hong Kong Research Grants Council under contract number HKU7124/99E.
}

the wireless channel is error-free. However, because the geographically scattered mobile users inevitably suffer from different degrees of fading and shadowing effects, indeed a common drawback of previous MAC protocols is that they assume the underlying physical layer always delivers a constant throughput, and as such, they may not be able to effectively utilize the precious bandwidth when the channel condition is swiftly varying among different users. In this paper, we propose a new MAC protocol that works closely with the underlying physical layer. As will be shown in this paper, the knowledge of the channel condition reported to the MAC layer by the physical layer is indeed a very useful component in achieving even higher performance in a wireless communication system where burst errors are the norm rather than exception.

Traditional physical layer is designed to deliver a constant throughput in that the amount of error protection incorporated into a packet is fixed without regard to the time varying channel condition. To exploit the time-varying nature of the wireless channel, a variable rate channel-adaptive physical layer is employed in our proposed protocol as illustrated in Figure 1(a). Channel state information (CSI), $c(t)$, which is estimated at the receiver, is fed back to the transmitter via a low-capacity feedback channel. Based on the CSI, the level of redundancy and the modulation constellation applied to the information packets are adjusted accordingly by choosing a suitable transmission mode. Thus, the instantaneous throughput is varied according to the instantaneous channel state in that more protection is added when the channel condition becomes worse. Using this dynamically adjusted level of protection, the targeted bit-error-rate (BER) is maintained. It has been shown [4] that a significant gain in the average throughput can be achieved in these adaptive channel 


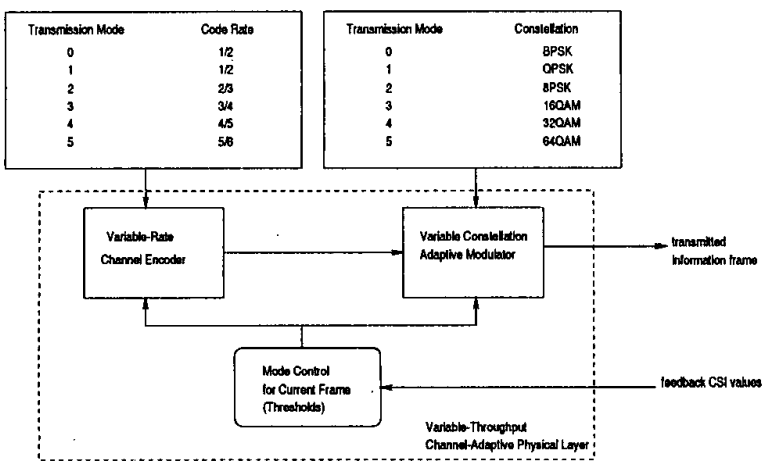

Fig. 1. A conceptual block diagram of the variable throughput channel adaptive physical layer.

coding schemes. Our major contribution in this paper is a new MAC layer protocol, called CHARISMA (CHannel Adaptive Reservation-based ISochronous Multiple Access), which is based on such a channel adaptive physical layer. CHARISMA is a dynamic TDMA (D-TDMA) based protocol with one major distinctive feature: the user contention requests are gathered by the base-station in the first phase of the time frame without immediately announcing the information slots assignment right after each contention mini-slot. After all requests are received, the information slots are assigned to the users based on their respective CSI ranking. Performance gain is derived by supplying an additional input to the MAC protocol, namely the channel state information (CSI) from the physical layer. We compare the performance of our proposed scheme with two baseline systems as shown in Figure 2. Baseline System-I is also a DTDMA based MAC layer but a constant throughput physical layer is used. In Baseline System-II [3], a variable rate adaptive physical layer is incorporated with a D-TDMA based MAC layer but there is no interaction between the two layers. Our extensive simulation results indicate that for the same quality of service level, CHARISMA achieves a considerably greater capacity.

\section{REsults}

In our simulation study, we assume a transmission bandwidth of $320 \mathrm{kHz}$ for the TDMA frames. Bit rate of the speech terminals is $8 \mathrm{kbps}$ which conforms to the values in GSM and CDMA systems. Table I summarizes the parameters we used.

The performance of packet loss probability versus the number of active voice user is shown in Figures 4 and 3. for the CHARISMA, System-I, and System-II. As can be seen, in all test cases System-II is superior than System-I in terms of the packet loss probability because it enjoys twice the offered throughput relative to System-I. This illustrates the merit of us-

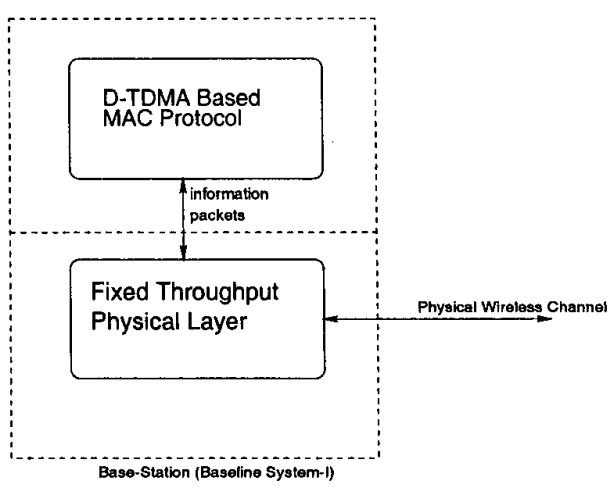

(a) baseline System-I (fixed-rate channel encoder)

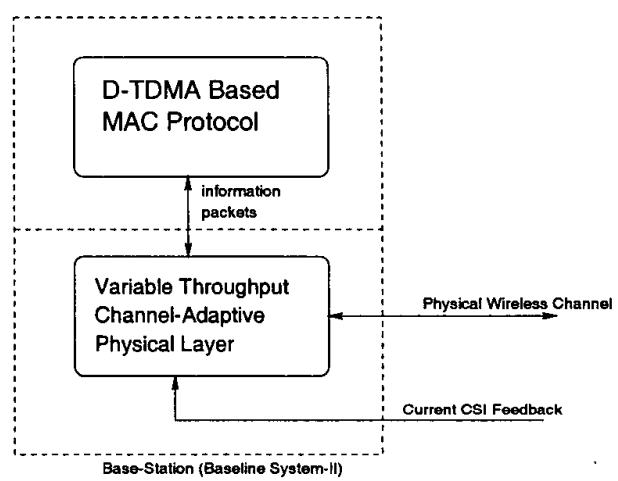

(b) baseline System-II (adaptive channel encoder)

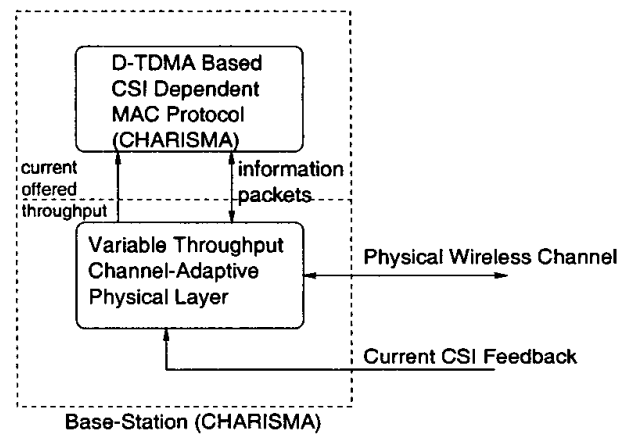

(c) CHARISMA (adaptive channel encoder with MAC interaction)

Fig. 2. Conceptual models of the physical and MAC layers. 
TABLE I

Simulation Parameters.

\begin{tabular}{ll}
\hline Parameter & Value \\
\hline number of request mini-slots $N_{r}$ & 13 \\
number of information slots $N_{i}$ & 8 \\
voice permission probability $p_{v}$ & 0.3 \\
data permission probability $p_{d}$ & 0.2 \\
data rate (data users) & $19.2 \mathrm{Kbps}$ \\
number of frames & $2 \times 10^{6}$ \\
\hline
\end{tabular}

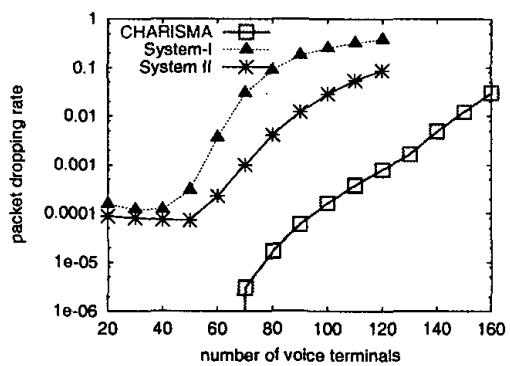

(a) $N_{d}=0$

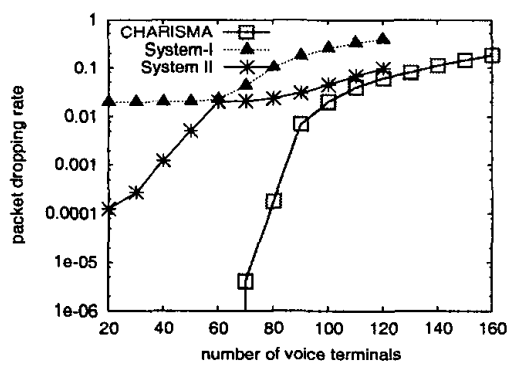

(b) $N_{d}=20$

Fig. 3. Voice terminals performance comparison for cases without capture.

ing a variable throughput physical layer. However, the potential of the CHARISMA protocol is demonstrated by the significant improvement in terms of packet dropping probability under the same traffic load of CHARISMA relative to System-II. For example, at the $1 \%$ voice packet dropping rate threshold [2], the number of voice terminals that can be accommodated by CHARISMA is 2.5 times and 1.87 times greater than those of System-I and System-II, respectively, for cases without capture and $N_{d}=0$ (see Figure 4(a)). For cases where there are data users, the CHARISMA protocol outperforms the two baseline systems by an even greater margin. These

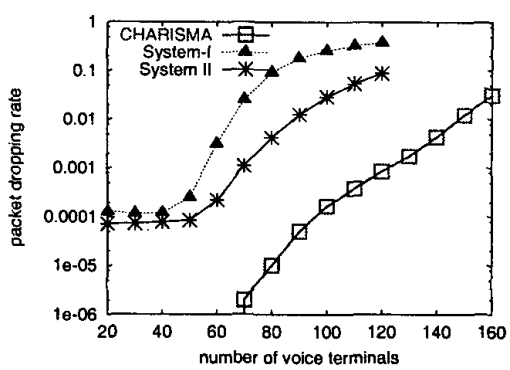

(a) $N_{d}=0$

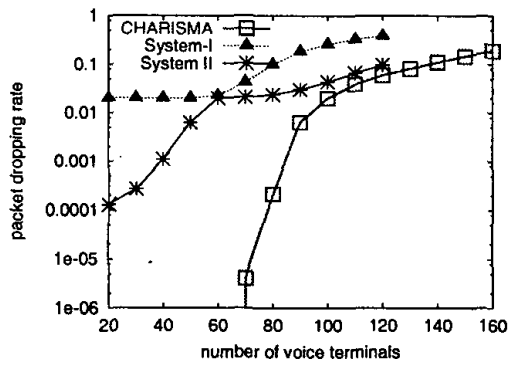

(b) $N_{d}=20$

Fig. 4. Voice terminals performance comparison for cases with capture.

results indicate that the capacity of the system is substantially increased by the intelligent interaction between the MAC and physical layers.

Figures 5 to 8 illustrate the performance of data terminal in terms of $\bar{\rho}$ and $\bar{D}_{d}$ respectively. When the traffic load is high, the system is in a highly congested state so that the average per-user throughput drops and the average per-user delay also increases dramatically. These adverse phenomena are detrimental to the data users' quality of service $(\mathrm{QoS})$, which depends critically on the parameters pair (delay, throughput). Again, in all test cases System-II is superior than System-I in terms of $\bar{\rho}$ and $\bar{D}_{d}$ because the former has twice the average offered throughput from the channel-adaptive physical layer. However, the remarkable effect of intelligent scheduling is again demonstrated by the performance gains of the proposed CHARISMA protocol relative to SystemII. For example, at a QoS level of (1 sec, 0.25$)$, the number of data users that can be accommodated by CHARISMA is 14 times and 2 times greater than those of System-I and System-II, respectively, for test cases without capture and $N_{v}=0$. Furthermore, for test cases with 20 voice users, the data users capacity of CHARISMA is 60 times and 2.2 times greater 
than those of System-I and System-II, respectively. The results with capture concur with these observations.

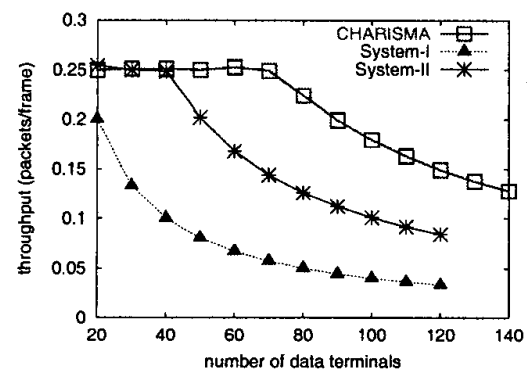

(a) $N_{v}=0$

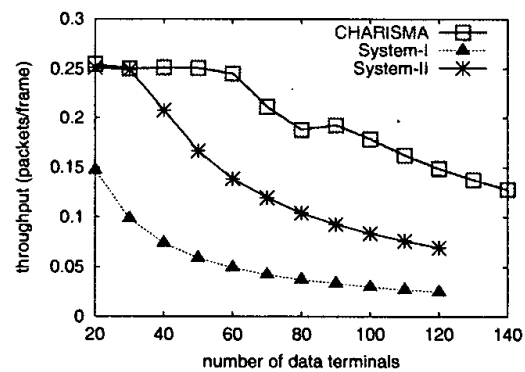

(b) $N_{v}=20$

Fig. 5. Data terminals performance-throughput against traffic load for cases without capture.

\section{A. Interpretations}

It is clearly demonstrated that, by the experiment results described above, the proposed CHARISMA protocol outperforms the two traditional approaches by a considerable margin. The substantial performance improvements are explicable by the following arguments.

- A voice terminal may experience a deep fading for a long time when it is affected by shadowing. In System-I or System-II, bandwidth allocation in the MAC layer is carried out regardless of the current channel condition as detected in the physical layer. Thus, information slots could also be allocated to such a user and the transmitted packets will be very likely lost due to the poor channel condition. In other words, assigned slots are simply wasted. This kind of wasteful allocation is avoided in our proposed CHARISMA protocol.

- Selection diversity is implicitly incorporated in the CHARISMA protocol. Through the priority based

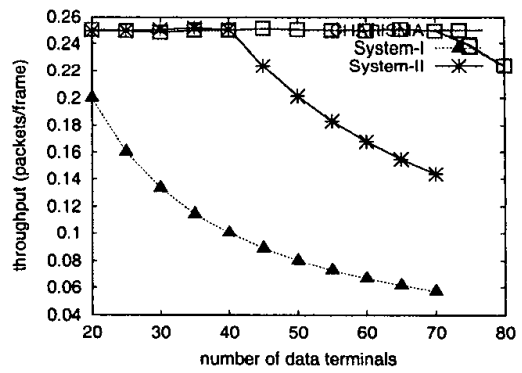

(a) $N_{v}=0$

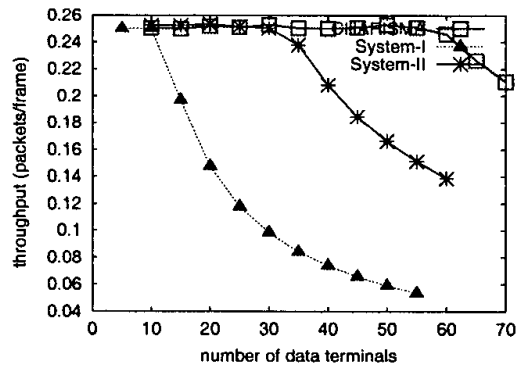

(b) $N_{v}=20$

Fig. 6. Data terminals performance-throughput against traffic load for cases with capture.

assignment process, every frame is packed with a selected group of information packets with good channel states. Thus, the effective delivered throughput per frame achieved in CHARISMA can be much higher than that in System-II. In CHARISMA, a large number of transmission requests are collected first before allocation of information slots. From the collection of requests, there is a high likelihood that a sufficient number of requests with good channel states can be selected to fully utilize the information slots in an effective manner (i.e., high throughput). For those requests with poor instantaneous channel states, their transmissions are deferred until when the CSI improves or the deadlines are approaching. By contrast, in System-II, requests are served in a first-come-first-serve manner due to the traditional strategy of immediately assigning slots upon successful receipt of requests. Thus, the channel states of such requests are highly diverse and, most importantly, some requests with bad channel states (hence very low throughput) are also served, whereby causing inefficient the bandwidth utilization. For example, a voice terminal may experience a very good CSI for a long time (out of shadowing). In System-I or System-II, this user, however, may fail to successfully 


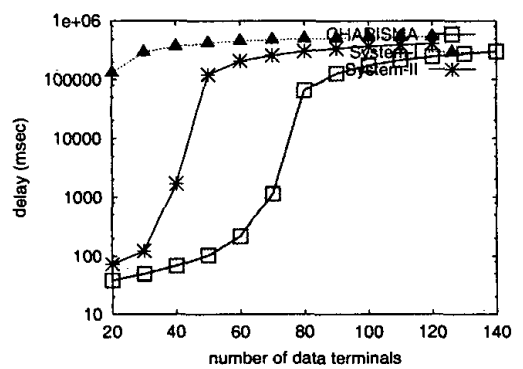

(a) $N_{v}=0$

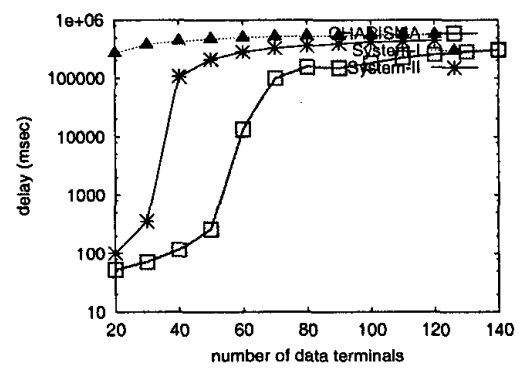

(b) $N_{v}=20$

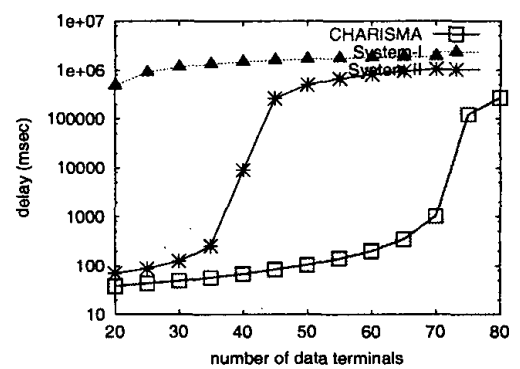

(a) $N_{v}=0$

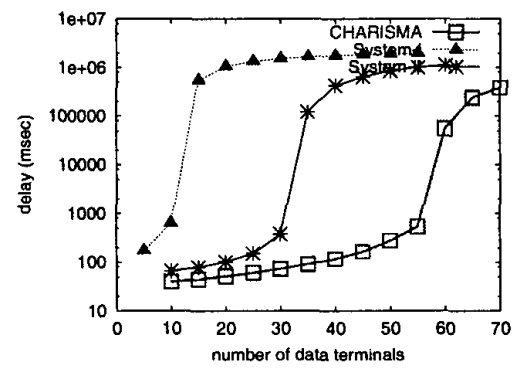

(b) $N_{v}=20$
Fig. 7. Data terminals performance-delay against traffic load for cases without capture.

transmitted a request to the base-station, probably because of excessive collisions in the request phase. In comparison, our proposed scheme gathers a large number of requests through successive frames, and allocate time slots to the users that can use the system bandwidth more effectively. Thus, the likelihood of "missing" a user with good channel state is much lower and the utilization of bandwidth is therefore higher.

- In general, the performance improvements with capture is slightly reduced compared to the situation without capture because the capture effect helps improving System-II. Specifically, a close scrutiny of the capture effect reveals that a request with a better CSI (i.e., higher power) is more likely to be captured than that of a request with a worse CSI (i.e., lower power). Thus, the capture process itself serves as a channel states selector-a request that has a higher throughput will be more likely to be chosen for slots assignment.

\section{REFERENCES}

[1] D. J. Goodman, R. A. Valenzuela, K. T. Gayliard, and B. Ramamurthi, "Packet Reservation Multiple Access for Local Wireless Communications," IEEE Transactions on
Fig. 8. Data terminals performance-delay against traffic load for cases with capture.

Communications, vol. 37, no. 8, pp. 885-890, Aug. 1989

[2] J. Gruber and L. Strawczynski, "Subjective Effects of Variable Delay and Speech Clipping in Dynamically Managed Voice Systems," IEEE Transactions on Communications, vol. COM-33, vol. 8, pp. 801-808, Aug. 1985.

[3] M. Kawagishi, S. Sampei, and N. Morinaga, "A Novel Reservation TDMA Based Multiple Access Scheme Using Adaptive Modulation for Multimedia Wireless Communication Systems," Proceedings of VTC'98, pp. 112-116, 1998.

[4] V. K. N. Lau and S. V. Maric, "Variable Rate Adaptive Modulation for DS-CDMA," IEEE Transactions on Communications, vol. 47, no. 4, pp. 577-589, April 1999. 